\title{
A Unifying Model of Variables and Names ${ }^{\star}$
}

\author{
Marino Miculan ${ }^{1}$ and Kidane Yemane $^{2}$ \\ 1 Dept. of Mathematics and Computing Science, \\ University of Udine, Via delle Scienze 206, I-33100 Udine, Italy \\ miculan@dimi.uniud.it \\ 2 Dept. of Information Technology, Uppsala University, \\ Box 337, S-751 05 Uppsala, Sweden \\ kidane.yemane@it.uu.se
}

\begin{abstract}
We investigate a category theoretic model where both "variables" and "names", usually viewed as separate notions, are particular cases of the more general notion of distinction. The key aspect of this model is to consider functors over the category of irreflexive, symmetric finite relations. The models previously proposed for the notions of "variables" and "names" embed faithfully in the new one, and initial algebra/final coalgebra constructions can be transferred from the formers to the latter. Moreover, the new model admits a definition of distinctionaware simultaneous substitutions. As a substantial application example, we give the first semantic interpretation of Miller-Tiu's $F O \lambda^{\nabla}$ logic.
\end{abstract}

\section{Introduction}

In recent years, many models for dynamically allocable entities, such as (bound) variables, (fresh) names, reference, etc., have been proposed. Most of (if not all) these models are based on some (sub)category of (pre)sheaves, i.e., functors from a suitable index category to Set $[19,6,10,8,5,18]$. The basic idea is to stratify datatypes according to various "stages" representing different degrees of information, such as number of allocated variables. A simple example is that of set-valued functors over $\mathbb{F}$, which is the category of finite subsets $C \subset \mathbb{A}$ of a given enumerable set $\mathbb{A}$ of abstract symbols ("variable names") $[6,10]$; here, the datatype of untyped $\lambda$-terms is the functor $\Lambda: \mathbb{F} \rightarrow$ Set, $\Lambda_{C}=\{t \mid F V(t) \subseteq C\}$. Morphisms between objects of the index category describe how we can move from one stage to the others; in $\mathbb{F}$, morphisms are any function $\sigma: C \rightarrow D$, that is any variable renaming possibly with unifications. Correspondingly, $\Lambda_{\sigma}: \Lambda_{C} \rightarrow \Lambda_{D}$ is the usual (capture-avoiding) variable renaming $-\{\sigma\}$ on terms.

Different index categories lead to different notions of "allocable entities". The notion of name, particularly important for process calculi, can be modeled using the subcategory $\mathbb{I}$ of $\mathbb{F}$ of only injective functions. Thus, stages of $\mathbb{I}$ can be still "enlarged" by morphisms (which corresponds to allocation of new names), but

\footnotetext{
* Work supported by EU projects IST-2001-33100 PROFUNDIS and IST-510996 TYPES.
} 
they cannot be "contracted", which means that two different symbols can never coalesce to the same. Categories of set- and domain-valued functors over II have been used for modeling $\pi$-calculus, $\nu$-calculus, etc. $[19,5]$.

According to this view, variables and names are quite different concepts, and as such they are rendered by different index categories. This separation is a drawback when we have to model calculi or logics where both aspects are present and must be dealt with at once. Some examples are: the fusion calculus, where names can be unified under some conditions; the open bisimulation of $\pi$-calculus, which is defined by closure under all (also unifying) distinction-preserving name substitutions; even, a (still unknown) algebraic model for the Mobile Ambients is supposed to deal with both variables and names (which are declared as different entities in capabilities); and finally, the logic $F O \lambda^{\nabla}[15]$, featuring a peculiar interplay between "global variables" and "locally scoped constants".

Why are $\mathbb{F}$ and $\mathbb{I}$ not sufficient to model these situations? The problem is that these models force the behaviour of atoms a priori. Atoms will always act as variables in $\mathbb{F}$, as names in $\mathbb{I}$. This is to be contrasted with the situations above, where the behaviour of an atom is not known beforehand.

A way for circumventing this problem is to distinguish allocation of atoms, from specifications of their behaviour. Behaviour of atoms is given a symmetric, irreflexive relation, called distinction: two atoms are related if and only if they cannot be unified, in any reachable stage. These relations can change dynamically, after that atoms are introduced. Thus a stage is a finite set of atoms, together with a distinction over it. These stages form the objects of a new index category $\mathbb{D}$, which subsumes both the idea of variables and that of names.

The aim of this paper is to give a systematic presentation of the model of set-valued functors over $\mathbb{D}$, first introduced by Ghani, Yemane and Victor for characterizing open bisimulation of $\pi$-calculus [9]. Following similar previous work $[6,5]$, we focus on algebraic, coalgebraic and logical properties of this category, relating these results with the corresponding ones in Set $^{\mathbb{F}}$ and $S e t^{\mathbb{I}}$.

In Section 2, we present the category $\mathbb{D}$, its properties and relations with $\mathbb{F}$ and $\mathbb{I}$. In Section 3 we study the structure of $S e t^{\mathbb{D}}$, and its relations with $S e t^{\mathbb{F}}$, Set ${ }^{\mathbb{I}}$. In particular, due to their importance for modeling process calculi, we will study initial algebras and final coalgebras of polynomial functors over Set $^{\mathbb{D}}$.

In Section 4, we give a general definition of the key notions of support and apartness, and then apply and compare their instances in the cases of Set $^{\mathbb{D}}$, $S e t^{\mathbb{F}}$ and $S e t^{\mathbb{I}}$. An application of apartness is in Section 5, where we present a monoidal definition of "apartness-preserving" simultaneous substitution.

In Section 6 we turn to the logical aspects of $S e t^{\mathbb{D}}$ : restricting to the subcategory of pullback-preserving functors, we define a self-dual quantifier similar to Gabbay-Pitts' $И$. This quantifier, and the structure of $S e t^{\mathbb{D}}$, will be put at work in Section 7 in giving the first denotational semantics of Miller-Tiu's $F O \lambda^{\nabla}$.

Final remarks and directions for future work are in Section 8.

Due to space limits, many proofs are omitted, but can be found in [14]. 


\section{Distinctions}

Let us fix an infinite, countable set of atoms $\mathbb{A}$. Atoms are abstract elements with no structure, intended to act both as variables and as names symbols.

We denote finite subsets of $\mathbb{A}$ as $n, m, \ldots$ Functions among these finite sets are "atom substitutions". The category of all these finite sets, and any maps among them is $\mathbb{F}$. The subcategory of $\mathbb{F}$ with only injective maps is $\mathbb{I}$. In fact, we can see a name essentially as an atom which must be kept apart from the others. We can formalize this concept as follows:

Definition 1. (The category $\mathbb{D}$ ) The category $\mathbb{D}$ of distinctions relations is the full subcategory of Rel of irreflexive, symmetric binary relations over $\mathbb{A}$ with a finite carrier set. (Here Rel is the category of relations and monotone functions.)

A distinction relation $(n, d)$ is thus a finite set $n$ of atoms and a symmetric relation $d \subseteq n \times n$ such that for all $i \in n:(i, i) \notin d$. In the following we will write $(n, d)$ as $d^{(n)}$, possibly dropping the superscript when clear from the context. A morphisms $f: d^{(n)} \rightarrow e^{(m)}$ is any monotone function $f: n \rightarrow m$, that is a substitution of atoms for atoms which preserves the distinction relation (if $(a, b) \in d$ then $(f(a), f(b)) \in e)$. In other words, substitutions cannot map two related (i.e., definitely distinct) atoms to the same atom of a later stage, while unrelated atoms can coalesce to a single one.

Structure of $\mathbb{D}$. The category $\mathbb{D}$ inherits from Rel products and coproducts. More explicitly, products and coproducts can be defined on objects as follows:

$$
\begin{aligned}
& d_{1}^{(m)} \times d_{2}^{(n)} \triangleq\left(m \times n,\left\{\left(\left(i_{1}, j_{1}\right),\left(i_{2}, j_{2}\right)\right) \mid\left(i_{1}, i_{2}\right) \in d_{1} \text { and }\left(j_{1}, j_{2}\right) \in d_{2}\right\}\right) \\
& d_{1}^{(m)}+d_{2}^{(n)} \triangleq\left(m+n, d_{1} \cup\left\{(l+i, l+j) \mid(i, j) \in d_{2}\right\}\right) \quad(l \triangleq \max (m)+1)
\end{aligned}
$$

where $m+n \triangleq m \cup\{l+i \mid i \in n\}$. Note that $\mathbb{D}$ has no terminal object, but it has initial object $(\emptyset, \emptyset)$. In fact, $\mathbb{D}$ inherits meets, joins and partial order from $\wp(\mathbb{A})$ :

$-d^{(n)} \wedge e^{(m)}=(d \cap e)^{(m \cap n)}$, and $d^{(n)} \vee e^{(m)}=(d \cup e)^{(m \cup n)}$

$-d^{(n)} \leq e^{(m)}$ iff $d \wedge e=d$, that is, iff $d \subseteq e$.

For each $n$, let us denote $\mathbb{D}_{n}$ the full subcategory of $\mathbb{D}$ whose objects are all relations over $n$. Then, $\mathbb{D}_{n}$ is a complete Boolean algebra. Let $\perp^{(n)} \triangleq(n, \emptyset)$ and $\top^{(n)} \triangleq\left(n, n^{2} \backslash \Delta_{n}\right)$ be the empty and complete distinction on $n$, respectively, where $\Delta: \mathbb{F} \rightarrow \operatorname{Rel}$ is the diagonal functor defined as $\Delta_{n}=(n,\{(i, i) \mid i \in n\})$.

$\mathbb{D}$ can be given another monoidal structure. Let us define $\oplus: \mathbb{D} \times \mathbb{D} \rightarrow \mathbb{D}$ as

$$
d_{1}^{(m)} \oplus d_{2}^{(n)}=\left(m+n, d_{1} \cup d_{2} \cup\{(i, j),(j, i) \mid i \in m, j \in n\}\right) .
$$

Proposition 1. ( $\left.\mathbb{D}, \oplus, \perp^{(0)}\right)$ is a symmetric monoidal category.

By applying coproduct and tensor to $\perp^{(1)}$ we get two distinguished dynamic allocation functors $\delta^{-}, \delta^{+}: \mathbb{D} \rightarrow \mathbb{D}$, as $\delta^{-} \triangleq \perp^{(1)}+{ }_{-}$and $\delta^{+} \triangleq \perp^{(1)} \oplus$. More explicitly, the action of $\delta^{+}$on objects is $\delta^{+}\left(d^{(n)}\right)=d_{+1}^{(n+1)}$ where $d_{+1}=$ $d \cup\{(*, i),(i, *) \mid i \in n\}$. Thus both $\delta^{-}$and $\delta^{+}$add an extra element to the carrier, but, as the superscript ${ }^{+}$is intended to suggest, $\delta^{+}$adds in extra distinctions. 
Embedding $\mathbb{I}$ and $\mathbb{F}$ in $\mathbb{D}$. Let $\mathbb{D}_{e}$ denote the full subcategory of $\mathbb{D}$ of empty distinctions $\perp^{(n)}=(n, \emptyset)$, and $\mathbb{D}_{c}$ the full subcategory of complete distinctions $\top^{(n)}=\left(n, n^{2} \backslash \Delta_{n}\right)$. Notice that all morphisms in $\mathbb{D}_{c}$ are mono morphisms of $\mathbb{D}$ - that is, injective maps.

Let us consider the forgetful functor $U: \mathbb{D} \rightarrow \mathbb{F}$, dropping the distinction relation. The functor $\mathrm{v}: \mathbb{F} \rightarrow \mathbb{D}_{e}$ mapping each $n$ in $\mathbb{F}$ to $\perp^{(n)}$, and each $f: n \rightarrow$ $m$ to itself, is inverse of the restriction of $U$ to $\mathbb{D}_{e}$.

On the other hand, the restriction of $U$ to $\mathbb{D}_{c}$ is a functor $U: \mathbb{D}_{c} \rightarrow \mathbb{I}$, because the only morphisms in $\mathbb{D}_{c}$ are the injective ones. The functor $t: \mathbb{I} \rightarrow \mathbb{D}_{c}$ mapping each $n$ in $\mathbb{I}$ to $\top^{(n)}$, and each $f: n \longmapsto m$ to itself, is inverse of $U$. Hence:

Proposition 2 . $\mathbb{D}_{e} \cong \mathbb{F}$, and $\mathbb{D}_{c} \cong \mathbb{I}$.

Therefore, we can say that the category of $\mathbb{D}$ generalises both $\mathbb{I}$ and $\mathbb{F}$. In fact, it is easy to check that the forgetful functor $U: \mathbb{D} \rightarrow \mathbb{F}$ is the right adjoint of the inclusion functor $\vee: \mathbb{F} \hookrightarrow \mathbb{D}$.

Remark 1. While we are on this subject, we define the functor $V: \mathbb{D} \rightarrow \mathbb{I}$ which singles out from each $d$ the (atoms of the) largest complete distinction contained in $d$. More precisely, $V$ is defined on objects as $V\left(d^{(n)}\right)=\max \left\{m \mid \top^{(m)} \leq d^{(n)}\right\}$ and on morphisms as the restriction. This defines a functor: if $f: d^{(n)} \rightarrow e^{(m)}$ is a morphism, then it preserves distinctions, and thus for $i \in V(d)$, since $i$ is part of a complete subdistinction of $d$, it must be mapped in a complete subdistinction of $e$, and hence $f(i) \in V(e)$. However, $V$ is not an adjoint of $\mathrm{t}$.

We recall finally that $\mathbb{F}$ has finite products (and hence also $\mathbb{D}_{e}$ ), while $\mathbb{I}$ has binary products only. Disjoint unions are finite coproducts in $\mathbb{F}$, but not in $\mathbb{I}$. Actually, disjoint union $\uplus: \mathbb{I} \times \mathbb{I} \rightarrow \mathbb{I}$ is only a monoidal structure over $\mathbb{I}$, which quite clearly corresponds to the restriction of $\oplus$ to $\mathbb{D}_{c}$ :

Proposition 3. $\oplus \circ\langle\mathrm{t}, \mathrm{t}\rangle=\mathrm{t} \circ \uplus$, that is, for $n, m \in \mathbb{I}: \top^{(n \uplus m)}=\top^{(n)} \oplus \top^{(m)}$. As a consequence, for Proposition 2, we have $\uplus=U \circ \oplus \circ\langle\mathrm{t}, \mathrm{t}\rangle$. On the other hand, $\oplus$ restricted to $\mathbb{D}_{e}$ is not equivalent to the coproduct + in $\mathbb{F}$.

\section{$3 \quad$ Presheaves over $\mathbb{D}$}

$S e t^{\mathbb{D}}$ is the category of functors from $\mathbb{D}$ to Set (often called presheaves (over $\left.\mathbb{D}^{o p}\right)$ ) and natural transformations. The structure of $\mathbb{D}$ lifts to Set $^{\mathbb{D}}$, which has: ${ }^{1}$

1. Products and coproducts, which are computed pointwise (as with all limits and colimits in functor categories); e.g. $(P \times Q)_{d^{(n)}}=P_{d^{(n)}} \times Q_{d^{(n)}}$. The terminal object is the constant functor $\mathcal{K}_{1}=\mathbf{y}\left(\perp^{(\emptyset)}\right): \mathcal{K}_{1}(d)=1$.

2. A presheaf of atoms Atom $\in S e t^{\mathbb{D}}$, Atom $=\mathbf{y}\left(\perp^{(1)}\right)=\mathbf{y}\left(\top^{(1)}\right)$. The action on objects is $\operatorname{Atom}\left(d^{(n)}\right)=n$.

${ }^{1}$ We shall use the same symbols for the lifted structure, but ensuring the reader has enough information to deduce which category we are working in. 
3. Two dynamic allocation functors $\delta^{-}, \delta^{+}: S e t^{\mathbb{D}} \rightarrow S e t^{\mathbb{D}}$, induced by each $\kappa \in\left\{\delta^{+}, \delta^{-}\right\}$on $\mathbb{D}$ as _ $\circ \kappa:$ Set $^{\mathbb{D}} \rightarrow$ Set $^{\mathbb{D}}$.

4. Let $\wp_{f}$ be the finite (covariant) powerset functor on Set; then $\wp_{f} \circ_{-}: S e t^{\mathbb{D}} \rightarrow$ Set $t^{\mathbb{D}}$ is the finite powerset operator on $\mathbb{D}$-presheaves.

5. Exponentials are defined as usual in functor categories:

$$
\begin{aligned}
\left(B^{A}\right)_{d} & \triangleq \operatorname{Set}^{\mathbb{D}}\left(A \times \mathbb{D}\left(d,,_{-}\right), B\right) \\
\left(B^{A}\right)_{f}(m) & \triangleq m \circ\left(i d_{A} \times\left({ }_{-} \circ f\right)\right) \text { for } f: d \rightarrow e \text { in } \mathbb{D}, m: A \times \mathbb{D}\left(d,_{-}\right) \longrightarrow B
\end{aligned}
$$

In particular, exponentials of representable functors have a nice definition:

Proposition 4. For all $d \in \mathbb{D}, B$ in $\operatorname{Set}^{\mathbb{D}}: B^{\mathbf{y}(d)} \cong B_{d+_{-}}$.

This allows us to point out a strict relation between Atom and $\delta^{-}$:

Proposition 5. (_) $)^{\text {Atom }} \cong \delta^{-}$, and hence ${ }_{-} \times$Atom $_{\text {}} \dashv \delta^{-}$.

Proof. Since Atom $=\mathbf{y}\left(\perp^{(1)}\right)$, by Proposition 4 we have that $F^{\text {Atom }} \cong F_{\perp^{(1)}+_{-}}=$ $F_{\delta^{-}(-)}=\delta^{-}(F)$. The second part is an obvious consequence.

The categories $S e t^{\mathbb{F}}$ and $S e t^{\mathbb{I}}$ can be embedded into $S e t^{\mathbb{D}}$.

Proposition 6. The functor $\vee: \mathbb{F} \hookrightarrow \mathbb{D}$ induces an essential geometric morphism $\vee: S e t^{\mathbb{F}} \rightarrow$ Set $t^{\mathbb{D}}$, that is two adjunctions $v_{!} \dashv v^{*} \dashv v_{*}$, where $v_{!} \cong \ldots U$, $\mathrm{v}^{*}={ }_{-} \circ \mathrm{v}$, and $\mathrm{v}_{*}(F)\left(d^{(n)}\right)=F_{n}$ if $d^{(n)}=\perp^{(n)}, 1$ otherwise.

Proof. The existence of the essential geometric morphism, and that the inverse image is _ov, is a direct application of [12-VII.2, Theorem 2]. Let us prove that $\mathrm{v}_{\mathrm{l}} \cong{ }_{-} \circ \bar{U} . \quad \mathrm{v}$ ! can be defined as the left Kan extension along $\mathbf{y}: \mathbb{F}^{o p} \hookrightarrow$ Set $^{\mathbb{F}}$ of the functor $T: \mathbb{F}^{o p} \rightarrow S e t^{\mathbb{D}}, T(n)=\mathbb{D}\left(\perp^{(n)}{ }_{-}\right)=\mathbf{y} \circ \vee^{o p}$. Hence:

$$
\begin{aligned}
\mathbf{v}_{!}(F) & =\left(\operatorname{Lan}_{\mathbf{y}}(T)\right)(F)=\int^{m \in \mathbb{F}} \operatorname{Set}^{\mathbb{F}}(\mathbf{y}(m), F) \cdot \mathbb{D}\left(\perp^{(m)},{ }_{-}\right) \\
& =\int^{m \in \mathbb{F}} F_{m} \cdot \mathbb{F}\left(m, U\left({ }_{-}\right)\right)=\left(\int^{m \in \mathbb{F}} F_{m} \cdot \mathbb{F}\left(m,{ }_{-}\right)\right) \circ U=F \circ U
\end{aligned}
$$

Proposition 7. v : Set $t^{\mathbb{F}} \rightarrow$ Set $^{\mathbb{D}}$ is an embedding, that is: $v^{*} \circ v_{*} \cong I d$.

As a consequence, by $[12-$ VII.4, Lemma 1$]$ we have also $v^{*} \circ v_{!} \cong I d$, and hence both $v_{*}$ and $v_{\text {! }}$ are full and faithful.

A similar result holds also for $\mathrm{t}: \mathbb{I} \hookrightarrow \mathbb{D}$, although the adjoints have not a neat description as in the previous case.

Proposition 8. $\mathrm{t}$ induces an essential geometric morphism $\mathrm{t}:$ Set $^{\mathbb{I}} \rightarrow$ Set $^{\mathbb{D}}$, that is two adjunctions $\mathrm{t}_{!} \dashv \mathrm{t}^{*} \dashv \mathrm{t}_{*}$, where for all $G: \mathbb{I} \rightarrow$ Set, and $d \in \mathbb{D}$, it is $\mathrm{t}_{*}(G)(d)=\operatorname{Set}^{\mathbb{I}}\left(\mathbb{D}\left(d, \mathrm{t}\left({ }_{-}\right)\right), G\right)$.

Proposition 9. $\mathrm{t}: S e t^{\mathbb{I}} \rightarrow S e t^{\mathbb{D}}$ is an embedding, that is: $\mathrm{t}^{*} \circ \mathrm{t}_{*} \cong I d$.

This means that also $t^{*} \circ t_{!} \cong I d$, and hence both $t_{*}$ and $t_{!}$are full and faithful. 
Algebras and Coalgebras of Polynomial Functors. It is well-known that any polynomial functor over Set (i.e., defined only by constant functors, finite products/coproducts and finite powersets) has initial algebra. This result has been generalized to $\operatorname{Set}^{\mathbb{F}}[6,10]$ in order to deal with signatures with variable bindings; in this case, polynomials can contain also Var, the functor of variables, and a dynamic allocation functor $\delta_{\mathbb{F}}: S e t^{\mathbb{F}} \rightarrow S e t^{\mathbb{F}}$. For instance, the datatype of $\lambda$-terms up-to $\alpha$-conversion can be defined as the initial algebra of the functor

$$
\Sigma_{\Lambda}(X)=\operatorname{Var}+X \times X+\delta_{\mathbb{F}}(X)
$$

A parallel generalization for dealing with name generation use the category $S e t^{\mathbb{I}}$ (and its variants) $[10,8,5]$, which provides the functor of names $N$ and a dynamic allocation functor $\delta_{\mathbb{I}}: S e t^{\mathbb{I}} \rightarrow S e t^{\mathbb{I}}$. The domain for late semantics of $\pi$-calculus [5] can be defined as the final coalgebra of the functor $B: S e t^{\mathbb{I}} \rightarrow S e t^{\mathbb{I}}$

$$
B P \triangleq \wp_{f}\left(N \times P^{N}+N \times N \times P+N \times \delta_{\mathbb{I}} P+P\right)
$$

In $S e t^{\mathbb{D}}$, we can generalize a step further. We say that a functor $F: S t^{\mathbb{D}} \rightarrow$ $S e t^{\mathbb{D}}$ is polynomial if it be defined by using only Atom, constant functors, finite products/coproducts, dynamic allocations $\delta^{+}$and $\delta^{-}$and finite powersets.

There is a precise relation among initial algebras of polynomial functors on $S e t^{\mathbb{F}}$ and $S e t^{\mathbb{D}}$. Let us recall a general result (see e.g. [10]):

Proposition 10. Let $\mathcal{C}, \mathcal{D}$ be two categories and $f: \mathcal{C} \longrightarrow \mathcal{D}, T: \mathcal{C} \longrightarrow \mathcal{C}$ and $T^{\prime}: \mathcal{D} \longrightarrow \mathcal{D}$ be three functors such that $T^{\prime} \circ f \cong f \circ T$ for some natural isomorphism $\phi: T^{\prime} \circ f \longrightarrow f \circ T$.

1. If $f$ has a right adjoint $f^{*}$, and $(A, \alpha: T A \rightarrow A)$ is an initial T-algebra in $\mathcal{C}$, then $\left(f(A), f(\alpha) \circ \phi_{A}: T^{\prime}(f(A)) \rightarrow f(A)\right)$ is an initial $T^{\prime}$-algebra in $\mathcal{D}$.

2. If $f$ has a left adjoint $f^{*}$, and $(A, \alpha: A \rightarrow T A)$ is a final T-coalgebra in $\mathcal{C}$, then $\left(f(A), \phi_{A}^{-1} \circ f(\alpha): f(A) \rightarrow T^{\prime}(f(A))\right)$ is a final $T^{\prime}$-coalgebra in $\mathcal{D}$.

For a polynomial functor $T: S e t^{\mathbb{D}} \rightarrow \operatorname{Set}^{\mathbb{D}}$, let us denote $\bar{T}: \operatorname{Set}^{\mathbb{F}} \rightarrow \operatorname{Set}^{\mathbb{F}}$ the functor obtained by replacing Atom with $\operatorname{Var}$ and $\delta^{+}, \delta^{-}$with $\delta_{\mathbb{F}}$ in $T$.

Theorem 1. The polynomial functor $T:$ Set $\mathbb{D}^{\mathbb{D}} \rightarrow$ Set ${ }^{\mathbb{D}}$ has initial algebra, which is (isomorphic to) $F \circ U$, where $(F, \alpha)$ is the initial $\bar{T}$-algebra in $S e t^{\mathbb{F}}$.

Proof. The functor $\bar{T}$ has initial algebra (see e.g. $[6,10]$ ); let us denote it by $(F, \alpha)$. In order to prove the result, we apply Proposition 10(1), where $f: \mathcal{C} \longrightarrow$ $\mathcal{D}$ is the functor $v_{!}={ }_{-} \circ U: S e t^{\mathbb{F}} \rightarrow S e t^{\mathbb{D}}$ of Proposition 6, whose right adjoint is $\mathrm{v}^{*}$. Then $\mathrm{v}_{!}(F)=F \circ U$. We have only to prove that $T \circ \mathrm{v}_{!} \cong \mathrm{v} ! \circ \bar{T}$. It is easy to see that this holds for products, coproducts, constant functors and finite powersets. It is also trivial to see that Atom $\cong V a r \circ U$.

It remains to prove that $\kappa \circ \mathrm{v} ! \cong \mathrm{v} ! \circ \delta_{\mathbb{F}}$, for $\kappa=\delta^{+}, \delta^{-}$. For $F$ a functor in $S e t^{\mathbb{F}}$, we prove that there is a natural isomorphism $\phi: \kappa\left(\mathrm{v}_{!}(F)\right)=\kappa(F \circ U) \longrightarrow$ $\mathrm{v}_{!}\left(\delta_{\mathbb{F}}(F)\right)=\delta_{\mathbb{F}}(F) \circ U$. This is trivial, because for $d^{(n)}$ a distinction in $\mathbb{D}$, it is $\kappa(F \circ U)_{d}=(F \circ U)_{\kappa d}=F_{U(\kappa d)}=F_{n+1}=\delta_{\mathbb{F}}(F)_{n}=\left(\delta_{\mathbb{F}}(F) \circ U\right)_{d}$.

Therefore, initial algebras of polynomial functors in $S e t^{\mathbb{D}}$ are exactly initial algebras of the corresponding functors in $S e t^{\mathbb{F}}$. This means that $S e t^{\mathbb{D}}$ can be used in place of $S e t^{\mathbb{F}}$ for defining datatypes with variable binding, as in e.g. [9].

There is a similar connection between $S e t^{\mathbb{I}}$ and $S e t^{\mathbb{D}}$, about final coalgebras. 
Lemma 1. $\delta^{+} \circ \mathrm{t}_{*} \cong \mathrm{t}_{*} \circ \delta_{\mathbb{I}}$ and $\delta^{-} \circ \mathrm{t}_{*} \cong \mathrm{t}_{*} \circ\left({ }_{-}\right)^{N}$.

Let $T: S e t^{\mathbb{I}} \rightarrow S e t^{\mathbb{I}}$ be a polynomial functor. Let us denote by $\tilde{T}: S e t^{\mathbb{D}} \rightarrow$ $S e t^{\mathbb{D}}$ the functor obtained by replacing in (the polynomial of) $T$, every occurrence of $N$ with $\mathrm{t}_{*}(N), \delta$ with $\delta^{+},\left({ }_{-}\right)^{N}$ with $\delta^{-}$. Then, we have the following:

Theorem 2. The functor $\tilde{T}: S e t^{\mathbb{D}} \rightarrow$ Set $^{\mathbb{D}}$ has final coalgebra, which is (isomorphic to $) \mathrm{t}_{*}(F)$, where $(F, \beta)$ is the final T-coalgebra in Set ${ }^{\mathbb{I}}$.

Therefore, in $S e t^{\mathbb{D}}$ we can define coalgebrically all the objects definable by polynomial functors in $S e t^{\mathbb{I}}$, like that for late bisimulation [5]. Moreover, Set $t^{\mathbb{D}}$ provides other constructors, such as Atom, which do not have a natural counterpart in $S_{e t}{ }^{\mathbb{I}}$. An example of application of these distinctive constructors, following [9], is the characterization of open semantics of $\pi$-calculus as the final coalgebra of the functor $B_{O}: S e t^{\mathbb{D}} \rightarrow S e t^{\mathbb{D}}:$

$$
B_{o} P \triangleq \wp_{f}\left(\text { Atom } \times \delta^{-} P+\text { Atom } \times \text { Atom } \times P+\text { Atom } \times \delta^{+} P+P\right)
$$

Notice that, although similar in shape, $B_{o}$ is not the lifting of the functor $B$ of strong late bisimulation in $\operatorname{Set}^{\mathbb{I}}$ (Equation 2), nor can be defined on $\operatorname{Set}^{\mathbb{I}}$.

\section{Support and Apartness}

A key feature of categories for modeling names is to provide some notion of support of terms/elements, and of non-interference, or "apartness" [19,8]. In this section, we first introduce a general definition of support and apartness, and then we examine these notions in the case of $S e t^{\mathbb{D}}$, and related categories.

Definition 2 (support). Let $\mathcal{C}$ be a category, $F: \mathcal{C} \rightarrow$ Set be a functor. Let $C$ be an object of $\mathcal{C}$, and $a \in F_{C} . A$ subobject $i: D \longmapsto C$ of $C$ supports $a($ at $C)$ if there exists a (not necessarily unique) $b \in F_{D}$ such that $a=F_{i}(b)$.

A support is called proper iff it is a proper subobject.

We denote by $\operatorname{Supp}_{F, C}(a)$ the set of subobjects of $C$ supporting $a$. The intuition is that $D$ supports $a \in F_{C}$ if $D$ is "enough" for defining $a$. It is clear that the definition does not depend on the particular subobject representative. As a consequence, $a$ is affected by what happens to elements in $D$ only:

Proposition 11. For all $D \in \operatorname{Supp}_{F, C}(a)$, and for all $h, k: C \rightarrow C^{\prime}:$ if $h_{\mid D}=$ $k_{\mid D}$ then $F_{h}(a)=F_{k}(a)$.

Notice that in general, the converse of Proposition 11 does not hold.

Remark 2. When $\mathcal{C}=\mathbb{F}, \mathbb{I}$, the supports of $a \in F_{n}$ can be seen as approximations at stage $n$ of the free variables/names of $a$-that is, the free variables/names which are observable from $n$. For instance, let us consider $t \in \Lambda_{n}$, where $\Lambda$ is the algebraic definition of untyped $\lambda$-calculus in equation 1 . It is easy to prove by induction on $t$ that for all $m \subseteq n: m \in \operatorname{Supp}_{\Lambda, n}(t) \Longleftrightarrow F V(t) \subseteq m$.

Supports are viewed as "approximations" because elements may have not any proper support, at any stage. For example, consider the presheaf Stream : 
$\mathbb{F} \rightarrow$ Set constantly equal to the set of all infinite lists of variables. The stream $s=\left(x_{1}, x_{2}, x_{3}, \ldots\right)$, which has infinite free variables, belongs to $S_{t r e a m_{n}}$ for all $n$, but also $\operatorname{Supp}_{\text {Stream }, n}(s)=\{n\}$.

$\operatorname{Supp}_{F, C}(a)$ is a poset, inheriting its order from $\operatorname{Sub}(a)$, and $C$ itself is always its top, but it may be that there are no proper supports, as shown in the remark above. Even in the case that an element has some finite (even proper) support, still it may be that it does not have a least support. (Consider, e.g., $G: \mathbb{F} \rightarrow$ Set such that $G_{n}=\emptyset$ for $|n|<2$, and $=\{x\}$ otherwise; then $x \in G_{\{x, y, z\}}$ is supported by $\{x, y\}$ and $\{x, z\}$ but not by $\{x\}$ alone.) However, we can prove the following:

Proposition 12. Let $\mathcal{C}$ have pullbacks, $F: \mathcal{C} \rightarrow$ Set be pullback-preserving, $C$ be in $\mathcal{C}$, and $x \in F_{C}$. If both $C_{1}, C_{2}$ support $x$ at $C$, then $C_{1} \wedge C_{2}$ supports $x$.

Remark 3. In the case that $\mathcal{C}=\mathbb{I}$, pullback-preserving functors correspond to sheaves with respect to the atomic topology, that is the Schanuel topos [12]. This subcategory of $S e t^{\mathbb{I}}$ has been extensively used in previous work for modeling names and nominal calculi; see $[10,4]$ among others, and ultimately also the FM techniques by Gabbay and Pitts $[8,17]$, since the category of nominal sets with finite support is equivalent to the Schanuel topos [8-Section 7].

We will use pullback-preserving functors over $\mathbb{D}$ in Section 6 below.

In the rest of the paper, we focus on the case when $\mathcal{C}$ is one of $\mathbb{F}, \mathbb{I}, \mathbb{D}$, which do have pullbacks and initial object $\left(\emptyset, \emptyset\right.$ and $\perp^{(\emptyset)}$ respectively). As one may expect, the support in $\mathbb{D}$ is a conservative generalization of those in $\mathbb{F}$ and $\mathbb{I}$ :

Proposition 13. 1. Let $n, m \in \mathbb{F}$, and $F: \mathbb{F} \rightarrow$ Set. For all $a \in F_{n}: m \in$ $\operatorname{Supp}_{F, n}(a) \Longleftrightarrow \mathrm{v}(m) \in \operatorname{Supp}_{\mathrm{v}_{1}(F), \mathrm{v}(n)}(a) .^{2}$

2. Let $n, m \in \mathbb{I}$, and $F: \mathbb{I} \rightarrow$ Set. For all $a \in F_{n}: m \in \operatorname{Supp}_{F, n}(a) \Longleftrightarrow \mathrm{t}(m) \in$ $\operatorname{Supp}_{t_{*}(F), \mathrm{t}(n)}(a)$.

We can now give the following general key definition, generalizing that used sometimes in $\operatorname{Set}^{\mathbb{I}}$ (see e.g. [19]).

Definition 3 (Apartness). Let $\mathcal{C}$ be a category with pullbacks and initial object. For $A, B: \mathcal{C} \rightarrow$ Set, the functor $A \#_{\mathcal{C}} B: \mathcal{C} \rightarrow$ Set ("A apart from $B$ ") is defined on objects as follows:

$$
\begin{aligned}
& \left(A \#_{\mathcal{C}} B\right)_{C}=\left\{(a, b) \in A_{C} \times B_{C} \mid \text { for all } f: C \rightarrow D:\right. \\
& \text { there exist } \left.s_{1} \in \operatorname{Supp}_{A, D}\left(A_{f}(a)\right), s_{2} \in \operatorname{Supp}_{B, D}\left(B_{f}(b)\right) \text { s.t. } s_{1} \wedge s_{2}=0\right\}
\end{aligned}
$$

For $f: C \rightarrow D$, it is $\left(A \#_{\mathcal{C}} B\right)_{f} \triangleq A_{f} \times B_{f}$.

As a syntactic shorthand, we will write pairs $(a, b) \in\left(A \#_{\mathcal{C}} B\right)_{c}$ as $a \# b$. In the following, we will drop the index $\mathcal{C}$ when clear from the context.

Let us now apply this definition to the three categories $S e t^{\mathbb{I}}, S e t^{\mathbb{F}}$, and $S e t^{\mathbb{D}}$.

\footnotetext{
${ }^{2}$ Recall that $\mathrm{v}_{!}(F)_{\mathrm{v}(n)} \cong F_{n}$, and hence it is consistent to consider $a \in \mathrm{v}_{!}(F)_{\mathrm{v}(n)}$.
} 
$\mathcal{C}=\mathbb{F}$ In this case we have that $a \# b$ iff at least one of $a, b$ is closed, i.e., it is supported by the empty set: if both $a$ and $b$ have only non-empty supports, then some variable can be always unified by a suitable morphism. So the definition above simplifies as follows:

$$
\left(A \#_{\mathbb{F}} B\right)_{n}=\left\{(a, b) \in A_{n} \times B_{n} \mid \emptyset \in \operatorname{Supp}_{A, n}(a) \text { or } \emptyset \in \operatorname{Supp}_{B, n}(b)\right\}
$$

$\mathcal{C}=\mathbb{I}$ In this case, names are subject only to injective renamings, and therefore can be never unified. So it is sufficient to look at the present stage, that is, the definition above simplifies as follows:

$$
\begin{aligned}
& \left(A \#_{\mathbb{I}} B\right)_{n}=\left\{(a, b) \in A_{n} \times B_{n} \mid\right. \\
& \text { there exist } \left.n_{1} \in \operatorname{Supp}_{A, n}(a), n_{2} \in \operatorname{Supp}_{B, n}(b) \text { s.t. } n_{1} \cap n_{2}=\emptyset\right\}
\end{aligned}
$$

which corresponds to say that $a \# b$ iff $a, b$ do not share any free name.

$\mathcal{C}=\mathbb{D}$ This case subsumes both previous cases: informally, $(a, b) \in(A \# B)_{d}$ means that if $i$ is an atom appearing free in $a$, then any $j$ occurring free in $b$ can never be unified with $i$, that is $(i, j) \in d$ :

$$
\begin{aligned}
& \left(A \#_{\mathbb{D}} B\right)_{d^{(n)}}=\left\{(a, b) \in A_{d} \times B_{d} \mid\right. \\
& \text { there exist } \left.s_{1} \in \operatorname{Supp}_{A, d}(a), s_{2} \in \operatorname{Supp}_{B, d}(b) \text { s.t. } s_{1} \oplus s_{2} \leq d\right\}
\end{aligned}
$$

Actually, all these tensors arise from the monoidal structures $\oplus$ and $\uplus$ of the categories $\mathbb{I}$ and $\mathbb{D}$, via the following general construction due to Day [3]:

Proposition 14. Let $(\mathcal{C}, \star, I)$ be a (symmetric) monoidal category. Then, $\left(\right.$ Set $\left.^{\mathcal{C}}, \star_{\mathcal{C}}, \mathbf{y}(I)\right)$ is a (symmetric) closed monoidal category, where

$$
\left(A \star_{\mathcal{C}} B\right)_{C}=\int^{C_{1}} A_{C_{1}} \times \int^{C_{2}} B_{C_{2}} \times \mathcal{C}\left(C_{1} \star C_{2}, C\right)
$$

Theorem 3. The monoidal structure $\left(\mathbb{D}, \oplus, \perp^{(\emptyset)}\right)$ induces, via equation 8 , the monoidal structure $\left(\operatorname{Set}^{\mathbb{D}}, \# \mathbb{D}, \mathbf{y}\left(\perp^{(0)}\right)=\mathcal{K}_{1}=1\right)$ of equation $\%$.

Proof. Let $A, B: \mathbb{D} \rightarrow$ Set, and $d^{(n)} \in \mathbb{D}$; by applying Proposition 14 and since products preserves coends, we have

$$
\begin{aligned}
\left(A \star_{\mathbb{D}} B\right)_{d} & =\iint_{d_{1}, d_{2}}^{d_{d_{1}}} A_{d_{2}} \times \mathbb{D}\left(d_{1} \oplus d_{2}, d\right) \\
& =\left(\coprod_{d_{1}, d_{2} \in \mathbb{D}} A_{d_{1}} \times B_{d_{2}} \times \mathbb{D}\left(d_{1} \oplus d_{2}, d\right)\right)_{/ \approx}
\end{aligned}
$$

where the equivalence $\approx$ is defined on triples as follows

$$
\begin{aligned}
\left(a, b, f: d_{1} \oplus d_{2} \rightarrow d\right) & \approx\left(a^{\prime}, b^{\prime}, g: d_{1}^{\prime} \oplus d_{2}^{\prime} \rightarrow d\right) \\
& \Longleftrightarrow A_{f \circ i n l}(a)=A_{g \circ i n l}\left(a^{\prime}\right) \text { and } B_{f \circ i n r}(b)=B_{g \circ i n r}\left(b^{\prime}\right)
\end{aligned}
$$


For each class $\left[\left(a, b, f: d_{1} \oplus d_{2} \rightarrow d\right)\right] \in\left(A \star_{\mathbb{D}} B\right)_{d}$ we can associate a unique pair $\left(A_{f \circ i n l}(a), B_{f \circ i n r}(b)\right) \in\left(A \#_{\mathbb{D}} B\right)_{d}$; the definition does not depend on the particular representative we choose.

On the converse, let us consider a pair $(a, b) \in\left(A \#_{\mathbb{D}} B\right)_{d}$; this means that

- there exists $f_{1}: s_{1} \longmapsto d, a^{\prime} \in A_{s_{1}}$ such that $a=A_{f_{1}}\left(a^{\prime}\right)$

- there exists $f_{2}: s_{2} \longmapsto d, b^{\prime} \in B_{s_{2}}$ such that $b=B_{f_{2}}\left(b^{\prime}\right)$

and such that $\left[f_{1}, f_{2}\right]: s_{1} \oplus s_{2} \longmapsto d$. We can associate this pair $(a, b)$ to the equivalence class of the triple $\left(a^{\prime}, b^{\prime},\left[f_{1}, f_{2}\right]\right)$ in the coend 9 . The class defined in this way does not depend on the particular $a^{\prime}$ and $b^{\prime}$ we choose.

It is easy to check that these two mappings are inverse of each other.

A similar constructions applies also to Set $^{\mathbb{I}}$, as observed e.g. in [19]:

Proposition 15. The monoidal structure $(\mathbb{I}, \uplus, 0)$ induces, via equation 8 , the monoidal structure $\left(\right.$ Set $\left.^{\mathbb{I}}, \#_{\mathbb{I}}, \mathbf{y}(0)=1\right)$ of equation 6 .

Using Theorem 3 , we can show that $\#_{\mathbb{F}}$ is a particular case of $\#_{\mathbb{D}}$ :

Proposition 16. $\#_{\mathbb{F}}=v^{*} \circ \#_{\mathbb{D}} \circ\left\langle v_{*}, v_{*}\right\rangle$.

Proof. Let us prove that for $F, G: \mathbb{F} \rightarrow$ Set, it is $\left(\mathrm{v}_{*}(F) \#_{\mathbb{D}} \vee_{*}(G)\right)_{\perp(n)} \cong$ $\left(F \#_{\mathbb{F}} G\right)_{n}$. By applying Theorem 3, we have

$$
\begin{aligned}
\left(\mathrm{v}_{*}(F) \#_{\mathbb{D}} \mathrm{v}_{*}(G)\right)_{\perp(n)} & =\left(\coprod_{d_{1}^{\left(n_{1}\right)}, d_{2}^{\left(n_{2}\right)} \in \mathbb{D}} \mathrm{v}_{*}(F)_{d_{1}} \times \mathrm{v}_{*}(G)_{d_{2}} \times \mathbb{D}\left(d_{1} \oplus d_{2}, \perp^{(n)}\right)\right)_{/ \approx} \\
& =\left(\coprod_{d_{1}^{\left(n_{1}\right)}, d_{2}^{\left(n_{2}\right)} \in \mathbb{D}} F_{n_{1}} \times G_{n_{2}} \times \mathbb{D}\left(d_{1} \oplus d_{2}, \perp^{(n)}\right)\right)_{/ \approx}
\end{aligned}
$$

Let us consider the set $\mathbb{D}\left(d_{1} \oplus d_{2}, \perp^{(n)}\right)$. If $d_{1} \oplus d_{2}=\perp^{(m)}$ for some $m$, then $\mathbb{D}\left(d_{1} \oplus d_{2}, \perp^{(n)}\right)=\mathbb{F}(m, n)$. Otherwise, $\mathbb{D}\left(d_{1} \oplus d_{2}, \perp^{(n)}\right)=\emptyset$.

Now, the only way for having $d_{1} \oplus d_{2}=\perp^{(m)}$ is that both $d_{1}$ and $d_{2}$ are empty relations $\perp^{\left(n_{1}\right)}, \perp^{\left(n_{2}\right)}$, and at least one of them has no atoms at all (otherwise the $\oplus$ would add a distinction in any case). Therefore, the equivalence above can be continued as follows:

$$
\ldots=\left(\left(\coprod_{n_{1} \in \mathbb{F}} F_{n_{1}} \times G_{\emptyset} \times \mathbb{F}\left(n_{1}, n\right)\right)+\left(\coprod_{n_{2} \in \mathbb{F}} F_{\emptyset} \times G_{n_{2}} \times \mathbb{F}\left(n_{2}, n\right)\right)\right)_{/ \approx}
$$

This means that the triples are either of the form $\left(a \in F_{\emptyset}, b \in G_{n_{2}}, f: n_{2} \rightarrow n\right)$, or of the form $\left(a \in F_{n_{1}}, b \in G_{\emptyset}, f: n_{1} \rightarrow n\right)$. The first is equivalent to the pair $\left(F_{?}(a), G_{f}(b)\right)$, the second to the pair $\left(F_{f}(a), G_{?}(b)\right)$, both in $\left(F \#_{\mathbb{F}} G\right)_{n}$.

The next corollary is a consequence of Theorem 3 and Proposition 14: 
Corollary 1. The functor $A \# \#_{-}:$Set $^{\mathbb{D}} \rightarrow$ Set $^{\mathbb{D}}$ has a right adjoint $[A]_{-}$, defined on objects by $([A] B)_{d}=\operatorname{Set}^{\mathbb{D}}\left(A, B_{d \oplus_{-}}\right)$.

Remark 4. Let us consider the counit $e v_{A, B}: A \#[A] B \longrightarrow B$ of this adjunction. For $d \in \mathbb{D}$, the component $e v_{d}:(A \#[A] B)_{d} \longrightarrow B_{d}$ maps an element $a \in A_{d}$ and

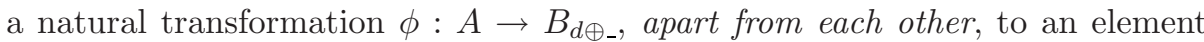
in $B_{d}$, which can be described as follows. Let $s_{1}, s_{2} \in \operatorname{Sub}(d)$ supporting $\phi$ and $a$, respectively, and such that $s_{1} \oplus s_{2} \leq d$. By the definition of support, let $\phi^{\prime}: A \rightarrow B_{s_{1} \oplus_{-}}$and $a^{\prime} \in A_{s_{2}}$ be the witnesses of $\phi$ and $a$ at $s_{1}$ and $s_{2}$, respectively. Then, $\phi_{s_{2}}^{\prime}\left(a^{\prime}\right) \in B_{s_{1} \oplus s_{2}}$, which can be mapped to an element in $B_{d}$ by the inclusion $s_{1} \oplus s_{2} \leq d$.

Finally, for $A=$ Atom we have the counterpart of Proposition 5:

Proposition 17. $[\text { Atom }]_{-} \cong \delta^{+}$, and hence \# Atom $_{\text {A }} \delta^{+}$.

\section{Substitution Monoidal Structure of $\boldsymbol{S e t}^{\mathbb{D}}$}

Let us define a tensor product $\bullet: S e t^{\mathbb{D}} \times S e t^{\mathbb{D}} \rightarrow S e t^{\mathbb{D}}$ as follows:

$$
\begin{aligned}
\text { for } A, B \in \text { Set }^{\mathbb{D}}: & A \bullet B \triangleq \int_{\text {that is, for } d}^{e \in \mathbb{D}} A_{e} \cdot B^{e} \\
\text { t } & (A \bullet B)_{d}=\int^{e \in \mathbb{D}} A_{e} \times\left(B^{e}\right)_{d}
\end{aligned}
$$

where, for $e^{(n)}$ in $\mathbb{D}, B^{e}: \mathbb{D} \rightarrow$ Set is the functor defined by

$$
\begin{aligned}
& \left(B^{e}\right)_{d}=\left\{\left(b_{1}, \ldots, b_{n}\right) \in\left(B_{d}\right)^{n} \mid \text { if }(i, j) \in e \text { then }\left(b_{i}, b_{j}\right) \in(B \# B)_{d}\right\} \\
& \left(B^{e}\right)_{f}=\left(B_{f}\right)^{n} \quad \text { for } f: d^{(m)} \rightarrow d^{\prime\left(m^{\prime}\right)}
\end{aligned}
$$

Unfolding the coend, we obtain the following explicit description of $A \bullet B$ :

$$
(A \bullet B)_{d}=\left(\coprod_{e \in \mathbb{D}} A_{e} \times\left(B^{e}\right)_{d}\right)_{/ \approx}
$$

where $\approx$ is the equivalence relation defined by

$$
\left(a ; b_{\rho(1)}, \ldots, b_{\rho(n)}\right) \approx\left(A_{\rho}(a) ; b_{1}, \ldots, b_{n^{\prime}}\right) \quad \text { for } \rho: e^{(n)} \rightarrow e^{\prime\left(n^{\prime}\right)}
$$

Actually, $B^{(-)}$can seen as a functor $B^{(-)}: \mathbb{D}^{o p} \rightarrow S e t^{\mathbb{D}}$, adding the "reindexing" action on morphisms: for $\rho: e^{(n)} \rightarrow e^{\prime\left(n^{\prime}\right)}$, define $B^{f}: B^{e^{\prime}} \longrightarrow B^{e}$ as the natural transformation with components $B_{d}^{f}:\left(B^{e^{\prime}}\right)_{d} \longrightarrow\left(B^{e}\right)_{d}, B_{d}^{f}\left(b_{1}, \ldots, b_{n^{\prime}}\right)=$ $\left(b_{f(1)}, \ldots, b_{f(n)}\right)$. It is easy to check that $B^{f}$ is well defined: if $(i, j) \in e^{\prime\left(n^{\prime}\right)}$, then $(f(i), f(j)) \in e^{(n)}$ and hence $\left(b_{f(i)}, b_{f(j)}\right) \in(B \# B)_{d}$. The functor $B^{(-)}$is a generalization of Cartesian extension; for instance, $B^{\perp^{(2)}}=B \times B, B^{\top^{(2)}}=B \# B$. 
We can give now a more abstract definition of _ $\bullet B:$ Set $^{\mathbb{D}} \rightarrow S e t^{\mathbb{D}}$, for all $B \in S e t^{\mathbb{D}}$. In fact, ${ }_{-} \bullet B$ arises as the left Kan extension of the functor $B^{(-)}$:

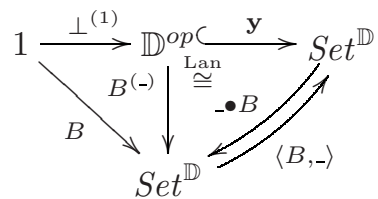

where $\left\langle B,{ }_{-}\right\rangle$is the right adjoint of $\_\bullet B$, defined as $\langle B, A\rangle_{d}=\operatorname{Set}^{\mathbb{D}}\left(B^{d}, A\right)$.

Proposition 18. (Set ${ }^{\mathbb{D}}, \bullet$, Atom) is a (non-symmetric) monoidal category .

Monoids in Set $^{\mathbb{D}}$ satisfy the usual properties of clones. In particular, the multiplication $\sigma: A \bullet A \rightarrow A$ of a monoid $(A, \sigma, v)$ can be seen as a distinctionpreserving simultaneous substitution: for every $d^{(n)} \in \mathbb{D}, \sigma_{d}$ maps (the class of) $\left(a ; a_{1}, \ldots, a_{m}\right) \in A_{e} \times\left(A^{e}\right)_{d}$ to an element in $A_{d}$, making sure that distinct atoms are "replaced by" elements which are apart (if $(i, j) \in e$, then $\left.\left(a_{i}, a_{j}\right) \in(A \# A)_{d}\right)$.

As in $[6,18]$, the monoidal structure of $\operatorname{Set}^{\mathbb{D}}$ can be used for characterizing presheaves coherent with apartness-preserving substitution; in particular, presheaves generated by binding signatures with constructors for distinctions, such as the signature of D-Fusion [2]. Details will appear elsewhere.

\section{Self-Dual Quantifier}

In this section we define a self-dual quantifier, in a suitable subcategory of $S e t^{\mathbb{D}}$. We begin with a standard construction of categorical logic. For $A, B \in \operatorname{Set}^{\mathbb{D}}$, let us consider the morphism $\theta: A \# B \hookrightarrow A \times B \stackrel{\pi}{\rightarrow} B$, given by inclusion in the cartesian product. We can define the inverse image of $\theta, \theta^{*}: \operatorname{Sub}(B) \rightarrow$ $\operatorname{Sub}(A \# B)$ : for $U \in \operatorname{Sub}(A)$, the subobject $\theta^{*}(U) \in \operatorname{Sub}(A \# B)$ is the pullback of $U \longmapsto B$ along $\theta$ : $\quad \theta^{*}(U)_{d}=\left\{(x, y) \in(A \# B)_{d} \mid y \in U_{d}\right\}$.

By general and well-known results $[16,12], \theta^{*}$ has both left and right adjoints, denoted by $\exists_{\theta}, \forall_{\theta}: \operatorname{Sub}(A \# B) \rightarrow \operatorname{Sub}(B)$, respectively. (If \# is replaced by $\times$, these are the usual existential and universal quantifiers $\exists, \forall: \operatorname{Sub}(A \times B) \rightarrow$ $\operatorname{Sub}(B)$.) Our aim is to prove that, under some conditions, it is $\exists_{\theta}=\forall_{\theta}$.

The condition is suggested by the following result, stating that if a property of a "well-behaved" type holds for a fresh atom, then it holds for all fresh atoms:

Proposition 19. Let $B: \mathbb{D} \rightarrow$ Set be a pullback preserving functor, and let $U$ a subobject of Atom $\#$ B. Let $d \in \mathbb{D}$, and $(a, x) \in U_{d}$. Then for all $b \in$ Atom $_{d}$ such that $b \# x:(b, x) \in U_{d}$.

Then, we have to restrict our attention to a particular class of subobjects:

Definition 4. Let $A: \mathbb{D} \rightarrow$ Set be an object of Set ${ }^{\mathbb{D}}$. A subobject $U \leq A$ is closed if for all $d \in \mathbb{D}, f: d \rightarrow e, x \in A_{d}$ : if $A_{f}(x) \in U_{e}$ then $x \in U_{d}$.

The lattice of closed subobjects of $A$ is denoted by $\operatorname{ClSub}(A)$. 
However, pullback-preserving subobjects of pullback-preserving functors are automatically closed, so this requirement is implied by the first one:

Proposition 20. Let $A: \mathbb{D} \rightarrow$ Set be a pullback preserving functor, and $U \leq A$ be a subobject of $A$. If also $U$ is pullback preserving, then it is closed.

Let us denote by $\mathcal{D}$ the full subcategory of $S e t^{\mathbb{D}}$ of pullback preserving functors. By above, for all $A \in \mathcal{D}$, the lattice $\operatorname{Sub}(A)$ of pullback-preserving subobjects is $\operatorname{ClSub}(A)$, but we will keep writing $\operatorname{ClSub}(A)$ for avoiding confusions.

For "well-behaved" types, $\theta^{*}$ restricts to closed subobjects:

Proposition 21. For all $A, B \in \mathcal{D}$ and $U \in \operatorname{ClSub}(A): \theta^{*}(U) \in \operatorname{ClSub}(A \# B)$.

Its left and right adjoints $\exists_{\theta}, \forall_{\theta}: \operatorname{ClSub}(A \# B) \rightarrow \operatorname{ClSub}(A)$ have the following explicit descriptions: for $U \leq A \# B$ :

$\exists_{\theta}(U)_{d}=\left\{y \in B_{d} \mid\right.$ there exist $f: d \rightarrow e, x \in A_{e}$, such that $x \# B_{f}(y)$ and $\left.\left(x, B_{f}(y)\right) \in U_{e}\right\}$

$\forall_{\theta}(U)_{d}=\left\{y \in B_{d} \mid\right.$ for all $f: d \rightarrow e, x \in A_{e}$, if $x \# B_{f}(y)$ then $\left.\left(x, B_{f}(y)\right) \in U_{e}\right\}$

Proposition 22. For all $B$ in $\mathcal{D}: \theta^{*} \circ \exists_{\theta}=i d_{\mathrm{ClSub}(\text { Atom\#B })}$

Proof. For $U \in \operatorname{ClSub}\left(\right.$ Atom \# B), we have to prove that $\theta^{*}\left(\exists_{\theta}(U)\right)=U$. Inclusion $\supseteq$ is trivial. Let us prove $\subseteq$. If $(a, y) \in \theta^{*}\left(\exists_{\theta}(U)\right)_{d}$, then $a \# y$, and by definition of $\exists_{\theta}$ there exist $f: d \rightarrow e, b \in$ Atom $_{e}$ such that $\left(b, B_{f}(y)\right) \in U_{e}$ (and hence $\left.b \# B_{f}(y)\right)$. But also $f(a) \# B_{f}(y)$, and therefore by Proposition 19, this means that also $\left(f(a), B_{f}(y)\right) \in U_{e}$. By closure of $U$, it must be $(a, y) \in U_{d}$.

Proposition 23. Let $B \in \mathcal{D}$, and $U \in \operatorname{ClSub}(B)$; then, for all $x \in U_{d}$, there exist $f: d \rightarrow e$ and $a \in$ Atom $_{e}$ such that $a \# B_{f}(x)$.

Proposition 24. For all $B$ in $\mathcal{D}: \exists_{\theta} \circ \theta^{*}=i d_{\mathrm{ClSub}(B)}$.

Proof. Let $U \in \operatorname{ClSub}(B)$ be a closed subobject. For any $d \in \mathbb{D}$, we have $\exists_{\theta}\left(\theta^{*}(U)\right)_{d}=\left\{x \in B_{d} \mid\right.$ there exist $f: d \rightarrow e, a \in$ Atom $_{e}$,

$$
\text { s.t. } \left.a \# B_{f}(x) \text { and }\left(a, B_{f}(x)\right) \in \theta^{*}(U)_{e}\right\}
$$

$=\left\{x \in B_{d} \mid\right.$ there exist $f: d \rightarrow e, a \in$ Atom $_{e}$, s.t. $a \# B_{f}(x)$ and $\left.B_{f}(x) \in U_{e}\right\}$

$=\left\{x \in U_{d} \mid\right.$ there exist $f: d \rightarrow e, a \in$ Atom $_{e}$, s.t. $\left.a \# B_{f}(x)\right\}$

For Proposition 23 above, this is exactly equal to $U_{d}$, hence the thesis.

Corollary 2. For $A \in \mathcal{D}$, the inverse image $\theta^{*}: \operatorname{ClSub}(A) \rightarrow \operatorname{ClSub}($ Atom $\# A)$ is an isomorphism, and hence $\theta^{*} \dashv \exists_{\theta}=\forall_{\theta} \dashv \theta^{*}$

Let us denote by $\boldsymbol{\eta}: \operatorname{ClSub}($ Atom $\# A) \rightarrow \operatorname{ClSub}(A)$ any of $\exists_{\theta}$ and $\forall_{\theta}$. There is a close connection between this quantifier and Gabbay-Pitts' (hence the notation); in fact, both quantifiers enjoy the following inclusions:

Proposition 25. Let $i: A \# B \hookrightarrow A \times B$ be the inclusion map, and $i^{*}$ : $\operatorname{ClSub}(A \times B) \rightarrow \operatorname{ClSub}(A \# B)$ its inverse image. Then: $\forall \leq \boldsymbol{И} \circ i^{*} \leq \exists$, that is, for all $U \in \operatorname{ClSub}(A \times B): \forall U \leq \boldsymbol{\eta}\left(i^{*}(U)\right) \leq \exists U$. 


\section{A Model for $F O \lambda^{\nabla}$}

In this section we apply the structure of $\mathcal{D}$ for giving a semantic interpretation of the logic $F O \lambda^{\nabla}[15] . F O \lambda^{\nabla}$ is a proof theory of generic judgments. Terms and typing judgments $\Sigma \vdash t: \tau$ of $F O \lambda^{\nabla}$ are as usual for simply typed $\lambda$-calculus, signatures $\Sigma$ are sets $x_{1}: \tau_{1}, \ldots, x_{m}: \tau_{m}$. Sequents have the form

$$
\Sigma: \sigma_{1} \triangleright B_{1}, \ldots, \sigma_{n} \triangleright B_{n} \longrightarrow \sigma_{0} \triangleright B_{0}
$$

where $\Sigma$ is the global signature, and each $\sigma_{i}$ is a local signature. A judgment $\sigma_{i} \triangleright B_{i}$ is called generic; each $B_{i}$ can use variables of the global signature $\Sigma$ or in the local signature $\sigma_{i}$ (formally: $\Sigma, \sigma_{i} \vdash B_{i}: o$ ). See [15] for further details.

Variable symbols in $F O \lambda^{\nabla}$ play two different roles. Those declared in global signatures act as variables of $\lambda$-calculus; instead, variables of local signatures act as "locally scoped constants", much like restricted names of $\pi$-calculus. A model of $F O \lambda^{\nabla}$ must account for both aspects at once, and this is the reason for neither $S e t^{\mathbb{F}}$ nor $S e t^{\mathbb{I}}$ (and their subcategories) can suffice. We can give an interpretation of both aspects in $\mathcal{D}$, taking advantage of its structure which subsumes those of $S e t^{\mathbb{F}}$ and $S e t^{\mathbb{I}}$ : as we will see, the dynamic allocation functor $\delta^{-}$, the apartness tensor (right adjoint to $\delta^{+}$) and the $\boldsymbol{U}$ quantifier will come into play.

The interpretation of types and terms is standard: each type $\tau$ is interpreted as a functor $\llbracket \tau \rrbracket$ in $\mathcal{D}$; the interpretation is extended to global signatures using the cartesian product. A well-typed term $\Sigma \vdash t: \gamma$ is interpreted as a morphism (i.e., a natural transformation) $\llbracket t \rrbracket: \llbracket \Sigma \rrbracket \longrightarrow \llbracket \gamma \rrbracket$ in $\mathcal{D}$. Notice that here, "local" signatures do not have any special rôle, so that terms are simply typed $\lambda$-terms without any peculiar "freshness" or "scoping" constructor. ${ }^{3}$

On the other hand, in the interpretation of generic judgments we consider variables in local signatures as distinguished atoms. A declaration $y$ appearing in a local signature $\sigma$, is intended as a "fresh, local" atom.

Remark 5. A correct model for $F O \lambda^{\nabla}$ would require a distinguished functor of atoms for each type (which can occur in local signatures) of the term language. Although it is technically possible to develop a typed version of the theory of $S_{e t} t^{\mathbb{D}}$ (along the lines of [13] for $S e t^{\mathbb{F}}$ ), it does not add anything substantial to our presentation; so in the following we assume variables of local signatures, or bound by $\nabla$, can be only of one type (denoted by $\alpha$ ). Hence, local signatures $\sigma$ are of the form $\left(y_{1}: \alpha, \ldots, y_{n}: \alpha\right)$, or better $\left(y_{1}, \ldots, y_{n}\right)$ leaving $\alpha$ 's implicit.

The distinguished type of propositions, $o$, is interpreted as the classifier of (closed) subobjects: $\llbracket o \rrbracket_{d}=\operatorname{ClSub}(\mathbf{y}(d))=\operatorname{ClSub}\left(\mathbb{D}\left(d,{ }_{-}\right)\right)$. A generic judgment $\left(y_{1}, \ldots, y_{n}\right) \triangleright B$ in $\Sigma$ (i.e., $\left.\Sigma, y_{1}: \alpha, \ldots y_{n}: \alpha \vdash B: o\right)$ is interpreted as a closed subobject $\llbracket\left(y_{1}, \ldots, y_{n}\right) \triangleright B \rrbracket_{\llbracket \Sigma \rrbracket} \leq \llbracket \Sigma \rrbracket$. More precisely, $\llbracket \sigma \triangleright B \rrbracket_{A} \in \operatorname{ClSub}(A)$ is defined first by induction on the length of the local context $\sigma$, and then by structural induction on $B$. Local declarations and the $\nabla$ quantifier are rendered by the functor $\boldsymbol{И}: \operatorname{ClSub}(A \# A$ tom $) \rightarrow \operatorname{ClSub}(A)$ above. Some interesting cases:

${ }^{3}$ As Miller and Tiu say, this is a precise choice in the design of $F O \lambda^{\nabla}$, motivated by the fact that standard unification algorithms still work unchanged. 


$$
\begin{array}{cc}
\llbracket(y, \sigma) \triangleright B \rrbracket_{A} \triangleq \mathbf{U}\left(\llbracket \sigma \triangleright B \rrbracket_{A \# A t o m}\right) & \llbracket \triangleright B_{1} \wedge B_{2} \rrbracket_{A} \triangleq \llbracket \triangleright B_{1} \rrbracket_{A} \wedge \llbracket \triangleright B_{2} \rrbracket_{A} \\
\llbracket \triangleright \nabla y \cdot B \rrbracket_{A} \triangleq \mathbf{U}\left(\llbracket \triangleright B \rrbracket_{A \# A t o m}\right) & \llbracket \triangleright \forall_{\gamma} x \cdot B \rrbracket_{A} \triangleq \forall\left(\llbracket \triangleright B \rrbracket_{A \times \llbracket \gamma \rrbracket}\right)
\end{array}
$$

It is easy to prove by induction on $\sigma$ that $\llbracket(\sigma, y) \triangleright B \rrbracket_{A}=\llbracket \sigma \triangleright \nabla y \cdot B \rrbracket_{A}$.

Finally, a sequent $\Sigma: \mathcal{B}_{1}, \ldots, \mathcal{B}_{n} \longrightarrow \mathcal{B}_{0}$ is valid if $\bigwedge_{i=1}^{n} \llbracket \mathcal{B}_{i} \rrbracket_{\llbracket \Sigma \rrbracket} \leq \llbracket \mathcal{B}_{0} \rrbracket_{\llbracket \Sigma \rrbracket}$. A rule $\frac{\mathcal{S}_{1} \ldots \mathcal{S}_{n}}{\mathcal{S}}$ is sound if, whenever all $\mathcal{S}_{1}, \ldots, \mathcal{S}_{n}$ are valid, also $\mathcal{S}$ is valid.

Using this interpretation, one can check that the rules of $F O \lambda^{\nabla}$ are sound. In particular, the rules $\nabla \mathcal{L}$ and $\nabla \mathcal{R}$ are trivial consequence of above. The verification of $\forall \mathcal{R}$, and $\exists \mathcal{L}$ requires some work. Here, we have to give a categorical account of a particular encoding technique, called raising, used to "gain access" to local constants from "outside" their scope. A simpler (i.e., monadic) application of raising occurs, in the following equivalence, which is provable in $F O \lambda^{\nabla}$ :

$$
\nabla x \forall_{\gamma} y \cdot B \equiv \forall_{\alpha \rightarrow \gamma} h \nabla x . B[(h x) / y] \quad \text { where } \Sigma, x: \alpha, y: \gamma \vdash B: o
$$

We show first how to represent (monadic) raising as in the equation 11; interestingly, it is here where the $\delta^{-}$comes into play. Referring to equation 11 , let us denote $A=\llbracket \Sigma \rrbracket$ and $C=\llbracket \gamma \rrbracket$. By the definition above, the interpretation of $B$ is a subobject of $(A \# A$ tom $) \times C$, while $B[(h x) / y]$ corresponds to a subobject of $\left(A \times C^{\text {Atom }}\right) \#$ Atom. Now, notice that $C^{\text {Atom }}=\delta^{-} C$ (Proposition 5$)$; thus, $h: \alpha \rightarrow \gamma$ is actually a term $\llbracket h \rrbracket \in \delta^{-} C$, that is a term which can make use of a locally declared variable. We can define the raising morphism

$$
\mathrm{r}:\left(A \times \delta^{-} C\right) \# \text { Atom } \rightarrow(A \# \text { Atom }) \times C \quad \text { mapping } \quad(x, h, a) \mapsto(x, a, h(a))
$$

The inverse image of $r$ is $r^{*}: \operatorname{ClSub}((A \#$ Atom $) \times C) \rightarrow \operatorname{ClSub}\left(\left(A \times \delta^{-} C\right) \#\right.$ Atom), defined by the following pullback:

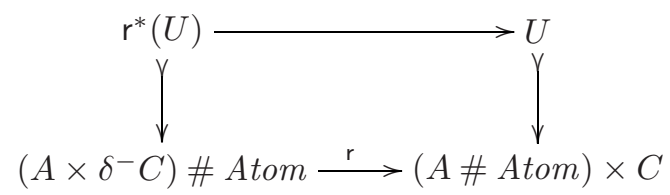

This morphism $r^{*}$ is the categorical counterpart of the syntactic raising:

Proposition 26. Let $\Sigma, x: \alpha, y: \gamma \vdash B:$ o. Let us denote $A=\llbracket \Sigma \rrbracket, C=\llbracket \gamma \rrbracket$. Then, $\mathrm{r}^{*}\left(\llbracket y \triangleright B \rrbracket_{C}\right)=\llbracket y \triangleright B[(h y) / x] \rrbracket_{A \times \delta^{-} C}$.

Then, quite obviously, the equation 11 states that $\boldsymbol{И} \circ \forall_{\gamma}=\forall_{\alpha \rightarrow \gamma} \circ \boldsymbol{И} \circ \boldsymbol{r}^{*}$, that is, the following diagram commutes:

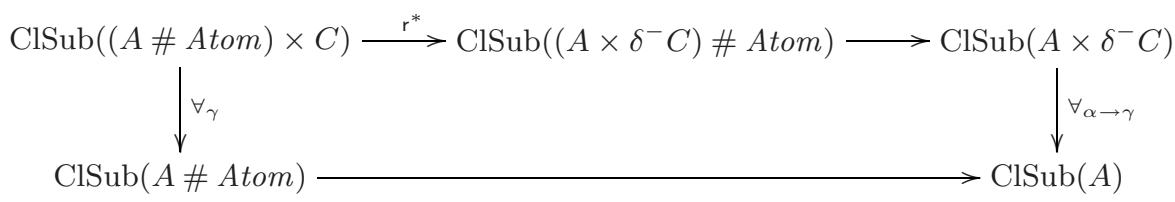

which can be checked by calculation. The raising morphism can be easily generalized to the polyadic case (recall that $B^{T^{(n)}}=B \# \cdots \# B, n$ times): 


$$
\begin{aligned}
\mathrm{r}:\left(A \times \delta^{-n} C\right) \# \text { Atom }^{\top^{(n)}} & \rightarrow\left(A \# \text { Atom }^{\top^{(n)}}\right) \times C \\
\left(x, h, a_{1}, \ldots, a_{n}\right) & \mapsto\left(x, a_{1}, \ldots, a_{n}, h\left(a_{1}, \ldots, a_{n}\right)\right)
\end{aligned}
$$

Then, the soundness of the rule $\forall \mathcal{R}$ is equivalent to the following:

Proposition 27. Let $A, C \in \mathcal{D}$ be functors, and $n \in \mathbb{N}$. Let $\pi: A \times \delta^{-n} C \rightarrow A$ be the projection, and $\mathrm{r}:\left(A \times \delta^{-n} C\right) \#$ Atom $^{T^{(n)}} \longrightarrow\left(A \#\right.$ Atom $\left.^{\top^{(n)}}\right) \times C$ the raising morphism. For all $G \in \operatorname{ClSub}(A)$, and $U \in \operatorname{ClSub}\left(\left(A \#\right.\right.$ Atom $\left.\left.^{T^{(n)}}\right) \times C\right)$, if $\pi^{*}(G) \leq \boldsymbol{И}^{n}\left(\mathbf{r}^{*}(U)\right)$ then $G \leq \boldsymbol{И}^{n}\left(\forall_{\gamma}(U)\right)$.

\section{Conclusions}

In this paper, we have studied a new model for dynamically allocable entities, based on the notion of distinction. Previous models for variables and for names can be embedded faithfully in this model, and also results about initial algebras/final coalgebras and simultaneous substitutions are extended to the more general setting. In a suitable subcategory of the model, it is possible to define also a self-dual quantifier, similar to Gabbay-Pitts' "И". This rich structure has allowed us to define the first denotational model for the logic $F O \lambda^{\nabla}$.

Future work. The rich structure of $S e t^{\mathbb{D}}$ can be useful also for modeling process calculi featuring both variables and names at once, like e.g. ambients. Actually, the intuition behind distinctions is also at the base of the D-Fusion calculus [2]; in fact, we think that the two binders $\lambda, \nu$ of D-Fusion can be modeled precisely by $\delta^{-}$and $\delta^{+}$in $S e t^{\mathbb{D}}$, respectively. Details will appear elsewhere.

$F O \lambda^{\nabla}$ is not complete with respect to the model presented in this paper: the И quantifier enjoys properties which are not derivable in $F O \lambda^{\nabla}$ (e.g., $\forall x . B \supset$ $\nabla x . B$ and $\nabla x . B \supset \exists x . B)$. One main reason is that $F O \lambda^{\nabla}$ does not admit weakening on local signature; for instance, the sequent $\Sigma: \sigma \triangleright B \longrightarrow(\sigma, y) \triangleright B$ is not derivable. This has been already noticed by Gabbay and Cheney, in their interpretation of $F O \lambda^{\nabla}$ into Fresh Logic [7], another first-order logic with a selfdual quantifier. Actually, we think that the $\boldsymbol{U}$ quantifier of $\mathcal{D}$ is closer to the $\boldsymbol{\Lambda}$ quantifier of Fresh Logic, than to the $\nabla$ of $F O \lambda^{\nabla}$. For this reason, it should be possible to model Fresh Logic in $\mathcal{D}$ quite easily - another future work.

Acknowledgments. The authors wish to thank Dale Miller and Alwen Tiu for useful discussions about $F O \lambda^{\nabla}$, and Neil Ghani for hints about Kan extensions.

\section{References}

1. J. Adamek, editor. Coalgebraic Methods in Computer Science, ENTCS. 2004.

2. M. Boreale, M. G. Buscemi, and U. Montanari. D-fusion: A distinctive fusion calculus. In Proc. APLAS'04, LNCS 3302, pages 296-310. Springer, 2004. 
3. B. J. Day. On closed categories of functors. In Reports of the Midwest Category Seminar, volume 137 of Lecture Notes in Mathematics, pages 1-38. Springer, 1970.

4. M. Fiore and S. Staton. Comparing operational models of name-passing process calculi. In Adamek [1].

5. M. Fiore and D. Turi. Semantics of name and value passing. In H. Mairson, editor, Proc. 16th LICS, pages 93-104, 2001.IEEE.

6. M. Fiore, G. Plotkin, and D. Turi. Abstract syntax and variable binding. In [11].

7. M. Gabbay and J. Cheney. A sequent calculus for nominal logic. In Proc. LICS'04, pages 139-148. IEEE Computer Society, 2004.

8. M. J. Gabbay and A. M. Pitts. A new approach to abstract syntax with variable binding. Formal Aspects of Computing, 13:341-363, 2002.

9. N. Ghani, K. Yemane, and B. Victor. Relationally staged computation in calculi of mobile processes. In Adamek [1].

10. M. Hofmann. Semantical analysis of higher-order abstract syntax. In Longo [11].

11. G. Longo, editor. Proc. 14th Symp. of Logic in Computer Science, 1999. IEEE.

12. S. Mac Lane and I. Moerdijk. Sheaves in Geometry and Logic. Springer, 1994.

13. M. Miculan and I. Scagnetto. A framework for typed HOAS and semantics. In Proc. PPDP'03, pages 184-194. ACM Press, 2003.

14. M. Miculan and K. Yemane. A unifying model of variables and names. TR UDMI/15/2004/RR, Dept. of Mathematics and Computing Science, Univ. of Udine, 2004. http://www.dimi.uniud.it/miculan/Papers/UDMI152004.pdf.

15. D. Miller and A. F. Tiu. A proof theory for generic judgments: An extended abstract. In LICS 2003, pages 118-127, 2003. IEEE.

16. A. M. Pitts. Categorical logic. In Handbook of LICS, vol. 5. OUP, 2000.

17. A. M. Pitts. Nominal logic, a first order theory of names and binding. Information and Computation, 186:165-193, 2003.

18. J. Power and M. Tanaka. Binding signatures for generic contexts. In Proc. TLCA'05, LNCS ?. Springer, 2005.

19. I. Stark. A fully abstract domain model for the $\pi$-calculus. In Proc. LICS'96. 